\title{
Reply to the letter "A proposal for a level for parapharyngeal extension of parotid gland"
}

\author{
Miquel Quer ${ }^{1}$
}

Received: 22 July 2016/Accepted: 26 July 2016/Published online: 29 July 2016

(C) Springer-Verlag Berlin Heidelberg 2016

\section{Dear Editor,}

Thank you very much for the opportunity to reply to the letter "A proposal for a level for parapharyngeal extension of parotid gland" to our paper "Classification of parotidectomies: a proposal of the European Salivary Gland Society" (ESGS). First of all, I would like to thank them for the favorable comments to our classification. We will considerer their suggestion to add a level for the parotid parapharyngeal extension and we will discuss in the next meeting of the ESGS. Of course our classification is open to improvements.

However, I see some limitations to their suggestion. Basically the main problems in my opinion are: (1) The ESGS classification is based on clearly anatomic landmarks and it is not easy to separate levels III-IV from the parapharyngeal extension of the parotid gland. (2) The resection of levels III-IV theoretically includes any parapharyngeal extension of the parotid gland, so I do not see a resection of levels III-IV preserving the parapharyngeal extension. (3) In the surgical treatment of parapharyngeal tumors originated in the parotid gland, the main discussion is the extent of resection of the parotid gland itself: how much parotid gland is it necessary to remove? Of course this depends on the pathology of the tumor and also in the extension so, depending on this, the surgeon will remove more or less parotid gland (levels III-IV or levels II-III-IV or I-II-III-IV). For this reason, the question is more the parotid itself than the parapharyngeal space extension.

Anyway I appreciate the comments and as I said, we will take this into consideration at the European Salivary Gland Society meetings.

This reply refers to the article available at doi:10.1007/s00405-016-4226-8.

Miquel Quer

mquer@santpau.cat;

http://www.orlsantpau.org

1 Department of Otorhinolaryngology and Head and Neck Surgery, Hospital de la Santa Creu i Sant Pau, Universitat Autònoma de Barcelona, Mas Casanovas, 90, 08041 Barcelona, Spain 